\title{
WQC - um Editor de WebQuest Colaborativo
}

\author{
Edjeane A. Nunes ${ }^{1}$, Lilian O. Ramires ${ }^{1}$ \\ ${ }^{1}$ Universidade Federal Rural de Pernambuco (UFRPE) - Unidade Acadêmica de Serra \\ Talhada (UAST) - Serra Talhada, PE - Brasil \\ edjeane.nunes@gmail.com, lilian.ramires@ufrpe.br
}

\begin{abstract}
Stimulating lessons and presenting content in a creative way is a challenge where educators need to face daily, requiring most of time, the usage of technological resources. However, the large amount of dubious and I or scientifically unproven origin content that are published indiscriminately in the network limits the usage of information technologies for school research aims. This way, WebQuest appears as an important tool to be enjoyed by the teacher and their students. Thus, the WQC-WebQuest Collaborative presented in this paper an online WebQuest editor that allows the creation and editing of WebQuests, tested by teachers and evaluated positively to perform this function.
\end{abstract}

Resumo. Dinamizar as aulas e apresentar os conteúdos de modo criativo é um desafio na rotina de educadores e, exige na maioria das vezes, o uso de recursos tecnológicos. Porém, a enorme gama de conteúdos de origem duvidosa elou sem comprovação científica, publicados de forma indiscriminada na rede limita o uso de Tecnologias da Informação para pesquisas no ambiente escolar. Desse modo, a WebQuest surge como uma importante ferramenta de direcionamento, a ser proposta pelo professor e utilizada pelos alunos. Assim, o WQC - WebQuest Collaborative apresentado neste trabalho é um editor de WebQuest on-line que permite a criação e a edição de WebQuests, testado com professores e avaliado de modo positivo para o que se propõe.

\section{Introdução}

Com o advento da tecnologia foram desenvolvidos diversos sistemas para facilitar tarefas antes impossíveis de serem realizadas. Em 1995 Bernie Dodge, professor da San Diego State University propôs aos alunos o desenvolvimento de uma tarefa de investigação usando principalmente recursos da Internet, a proposta metodológica era a de uma atividade orientada e investigativa de maneira que o assunto abordado fosse exposto de forma criativa [Silva e Ferrari 2006], surgiu então a WebQuest (WQ).

No âmbito da educação a WebQuest atua como uma metodologia mediadora no processo ensino-aprendizagem, onde o educador desenvolve e apresenta ao grupo de aprendizes uma atividade prática sobre determinado conteúdo, sendo utilizada a web para a apresentação da atividade e da pesquisa, que deve ser realizada em grupo e de forma colaborativa. Essa metodologia tem crescido e atingido cada vez mais educadores que procuram dinamizar suas aulas, estimular o trabalho em equipe, bem como difundir o uso de tecnologias na educação [Silva e Ferrari 2006]. Uma WQ envolve o uso da 
V Congresso Brasileiro de Informática na Educação (CBIE 2016)

Anais do XXVII Simpósio Brasileiro de Informática na Educação (SBIE 2016)

colaboração no desenvolvimento de tarefas realizadas em sala de aula baseado na convicção de que "aprendemos mais e melhor com os outros do que sozinhos" [Dodge 2003].

Algumas teorias de aprendizagem associadas à WebQuest a tornam ainda mais relevante no que se refere a atividades colaborativas. A teoria sócio-interacionista, por exemplo, tem muito a contribuir nesse aspecto. Vygotsky (1989) nessa teoria cita a aprendizagem como "[...] um processo social no qual os sujeitos constroem seus conhecimentos através da sua interação com o meio e com os outros [...]". Desse modo, a WebQuest media o processo ensino-aprendizagem e lança mão do uso de trabalhos colaborativos como forma de sobrepor as dificuldades no referido processo e de abreviar resultados positivos nesse sentido.

A utilização dessa atividade em sala de aula surge como uma alternativa didática para educadores, pois, inovar nas aulas é uma necessidade constante na rotina de professores e, conseguir desenvolver sempre um modo criativo de apresentar os conteúdos é um desafio que exige na maioria das vezes o uso da tecnologia e de ferramentas inovadoras. Segundo Neto et al. (2014), alguns educadores têm dificuldade em utilizar recursos tecnológicos e/ou desenvolver páginas eletrônicas, então a construção de um editor fácil de usar permite ajudá-los a criar atividades de maneira que a apresentação dos conteúdos seja mais atraente tanto para os alunos, quanto para o professor e sem exigir muito conhecimento prático e teórico dos usuários desse sistema.

Dessa maneira, este trabalho visa apresentar um editor de WebQuest, chamado WQC - WebQuest Collaborative, para que o professor possa propor aos alunos uma atividade de pesquisa realizada de modo coletivo, pregando objetividade e clareza em sua composição e guiando os alunos durante o processo de investigação a partir de fontes de pesquisa seguras. Com isso, fornecendo para os professores uma interface simples e uma área de edição com ferramentas semelhantes às encontradas nos editores de texto mais comuns, aumentando a praticidade no processo de formatação, reduzindo a complexidade na confecção das WebQuests e por consequência o trabalho dos autores. Outro fator a ser considerado é a existência de poucos sistemas semelhantes a esse, disponíveis de forma gratuita e principalmente com interfaces usáveis e em português.

\section{Referencial Teórico}

\subsection{WebQuest}

Conforme Bottentuit Junior e Coutinho (2010) o termo WebQuest etimologicamente parte da soma de duas palavras, Web (rede de hiperligações) e Quest (questionamento, busca ou pesquisa). Segundo Dodge (1995), WebQuest é uma investigação orientada na qual algumas ou todas as informações com as quais os aprendizes interagem são originadas de recursos da Internet, opcionalmente suplementadas com videoconferências, chats, fóruns, correio eletrônico e uso de outros meios para produção de escritas colaborativas, tais como wiki, blogs, etc.

Bottentuit Junior e Coutinho (2010) definem a Internet como um enorme conglomerado de informações de acesso fácil e rápido, disponíveis em diversos formatos e nas mais variadas fontes, em constante mutação e com um crescimento exponencial. Desse modo, conforme Silva e Ferrari (2009), a WebQuest apresenta como 
V Congresso Brasileiro de Informática na Educação (CBIE 2016)

Anais do XXVII Simpósio Brasileiro de Informática na Educação (SBIE 2016)

metodologia uma atividade de forma atrativa e com um direcionamento definido a partir do assunto abordado. Assim, essa técnica evita a chamada "surfagem" pela rede sem uma tarefa clara em mente e coíbe o plágio, aumentando a produtividade dos alunos por meio da especificação de diretrizes a serem seguidas e fazendo a recomendação de referências bibliográficas.

Segundo Bottentuit Junior e Coutinho (2010) faz-se necessária uma reflexão por parte dos educadores em relação à utilização dos recursos da web pelos discentes, considerando estratégias que levem os alunos a converterem informação em conhecimento e não que mecanizem a reprodução dos conteúdos encontrados na rede. Logo, as táticas de pesquisas orientadas na web, assumem um relevante grau de importância no processo ensino-aprendizagem, principalmente devido a enorme gama de conteúdos de origem duvidosa e/ou sem comprovação científica publicados de forma indiscriminada. Assim, a WebQuest surge como uma ferramenta de direcionamento a ser proposta pelo professor e utilizada pelos alunos como suporte à pesquisa.

A WebQuest pode auxiliar a construção de atividades embasadas na teoria construcionista, que encara o computador como um instrumento mediador para que o aluno construa o conhecimento. Essa corrente permite estabelecer relações que surgem pela interação e participação de seus membros em atividades coletivas. De acordo com Dodge (2003), toda WebQuest prevê tarefas em grupos, e esses grupos trabalham em prol do alcance de um único objetivo. A tarefa proposta pelos educadores deve possuir um cunho investigativo e a solução partir do trabalho colaborativo de cada equipe formada. Pois, o real intuito da metodologia adotada é incentivar equipes a trabalharem totalmente de forma colaborativa, deixando de lado a competitividade para buscar resultados sempre coletivamente e não apenas de modo cooperativo, ou seja, todos os integrantes desenvolvem juntos a solução e não apenas uma fração dela.

Essa atividade é estruturalmente composta por sete partes, sendo elas: introdução, tarefa, recursos, processo, avaliação, conclusão e créditos. Respectivamente o grupo tem uma breve introdução do tema abordado; seguido pela atividade a ser realizada; os recursos sugeridos (fontes de pesquisa na web aprovadas pelo professor); as etapas de desenvolvimento do trabalho que devem utilizar os recursos; o processo avaliativo ao qual o grupo será submetido; a importância de estudar o referido tema; e finalmente as referências utilizadas pelo professor durante o desenvolvimento da WebQuest.

\subsection{Trabalhos Relacionados}

O trabalho de Vieira (2008) apresenta o desenvolvimento da ferramenta Webquester. Esta permite que um professor, usando um navegador, crie sites dinâmicos e realize avaliações on-line a partir de templates pré-programados conforme um WebQuest.

O trabalho desenvolvido por Camargo e Fernandes (2010) apresenta a construção do editor de WebQuest WQE, um acrônimo para WebQuest Editor, que permite gerar WebQuests no formato do padrão IMS Learning Design - IMSLD (do inglês Instructional Management System Learning Design), um padrão que contém uma metalinguagem para construção de unidades de aprendizado, livre de métodos pedagógicos [IMS 2003], de modo a facilitar a criação de WebQuests no contexto de 
V Congresso Brasileiro de Informática na Educação (CBIE 2016)

Anais do XXVII Simpósio Brasileiro de Informática na Educação (SBIE 2016)

Sistemas de Gerência de Aprendizagem - LMS (do inglês Learning Management Systems). O WQE pode ser usado também fora do contexto de LMS, no formato HTML.

Na Tabela 1 é possível observar uma análise comparativa desses trabalhos em relação a este editor construído, a análise expõe tanto os pontos correspondentes, quanto os pontos distintos.

Tabela 1. Análise comparativa dos trabalhos relacionados

\begin{tabular}{|c|c|c|}
\hline \multirow{2}{*}{$\begin{array}{c}\text { TRABALHOS } \\
\text { RELACIONADOS }\end{array}$} & \multicolumn{2}{|l|}{ CARACTERÍSTICAS } \\
\hline & CORRELAÇÃO & DISTINÇÕES \\
\hline $\begin{array}{l}\text { Webquester: Ferramenta para } \\
\text { Criação de WebQuest com } \\
\text { Avaliações On-Line. }\end{array}$ & $\begin{array}{l}\text { - Padrão Bernie Dodge; } \\
\text { - Uso de WQ em salas de aula; } \\
\text { - Desenvolvimento utilizando de CSS, } \\
\text { jQuery, MySQL e PHP; } \\
\text { - WebQuests disponibilizadas em HTML. }\end{array}$ & $\begin{array}{l}\text { - Possui avaliações } \\
\text { on-line. }\end{array}$ \\
\hline $\begin{array}{l}\text { WQE: um Editor de } \\
\text { WebQuests Versátil. }\end{array}$ & $\begin{array}{l}\text { - Padrão Bernie Dodge (adota dois } \\
\text { modelos); } \\
\text { - Uso de WQ em salas de aula; } \\
\text { - WebQuests disponibilizadas em HTML. }\end{array}$ & $\begin{array}{l}\text { - Padrão Bernie } \\
\text { Dodge adaptado; } \\
\text { - Segue especificações } \\
\text { IMSLD. }\end{array}$ \\
\hline
\end{tabular}

\section{Metodologia}

O projeto é desenvolvido em cinco etapas executadas de forma incremental: estudo do tema, levantamento de requisitos, análise, implementação e testes de validação. A etapa inicial é necessária para conhecer o tema. O levantamento de Requisitos Funcionais (RF) e de Requisitos Não Funcionais (RNF) é realizado com a colaboração de um professor consultor em conjunto com o desenvolvedor. Partindo de tais requisitos, a etapa de análise é iniciada, onde o sistema é moldado a partir dos diagramas de Casos de Uso e de Componentes, atendendo as especificações definidas anteriormente.

O passo seguinte é a implementação, onde são utilizados o HTML5 (HyperText Markup Language), uma linguagem para estruturação e apresentação de conteúdo, o CSS3 (Cascading Style Sheets), mecanismo simples onde se define estilos para páginas web, a Linguagem de Programação PHP, que permite criar sites web dinâmicos, o plugin CKEditor, um editor de texto HTML pronto para usar e o sistema de gerenciamento de banco de dados MySQL.

Por fim, a fase de validação do sistema, realizada a partir da coleta de dados em uma amostra composta por 5 educadores. Onde, em um primeiro momento os professores são submetidos a uma aula expositiva sobre o tema WebQuest, seguida por uma etapa de cunho prático, em que a amostra citada anteriormente utiliza o editor de WebQuest WQC e responde a um questionário.

\section{WQC - WebQuest Collaborative}

O editor de WebQuest WQC - WebQuest Collaborative foi desenvolvido na plataforma web. A ferramenta possibilita a criação, a edição e a remoção de WebQuests em cada sessão iniciada, permitindo também que cada usuário, através de uma busca, consiga gerar uma lista com as WebQuests salvas por ele no banco de dados. 
V Congresso Brasileiro de Informática na Educação (CBIE 2016)

Anais do XXVII Simpósio Brasileiro de Informática na Educação (SBIE 2016)

Conforme se pode observar na Figura 1, o editor apresenta em sua Tela Inicial a explicação da metodologia, uma área para acesso ao sistema, um menu composto por uma aba CADASTRO e outra AJUDA. Clicando em CADASTRO se tem acesso a tela em que o professor no primeiro uso preenche seus dados (nome, e-mail e senha) para acessar o sistema. O sistema fornece apenas um tipo de permissão, ou seja, todos os usuários podem realizar todas as operações permitidas pelo software e a partir da efetuação do cadastro o visitante passa a ser usuário da ferramenta e ter total acesso por meio do fornecimento de um login e uma senha, válidos e previamente cadastrados. E na opção AJUDA ele tem explicações sobre o acesso ao sistema.

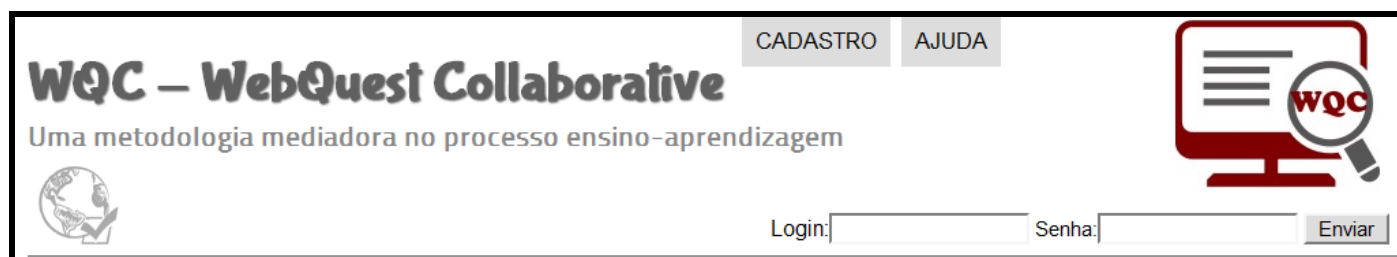

WebQuest > Home

Construa sua WebQuest

por Edjeane Alves

O que é

O termo WebQuest foi definido em 1995 por Bernie Dodge professor da Universidade de San Diego State University (SILVA: FERRARI, 2009) e conforme Bottentuit Junior e Coutinho (2010) etimologicamente o termo parte da soma de duas palavras, Web (rede de hiperligações) e Quest (questionamento, busca ou pesquisa). Segundo Dodge (1996) WebQuest é uma investigação orientada na qual algumas ou todas as informações com as quais os aprendizes interagem são originadas de recursos da Internet, opcionalmente suplementadas com videoconferências, chats, fóruns, correio eletrônico e uso de outros meios para produção de escritas colaborativas, tais como wiki, blogs, etc.

Figura 1. Tela para Login no WQC - WebQuest Collaborative

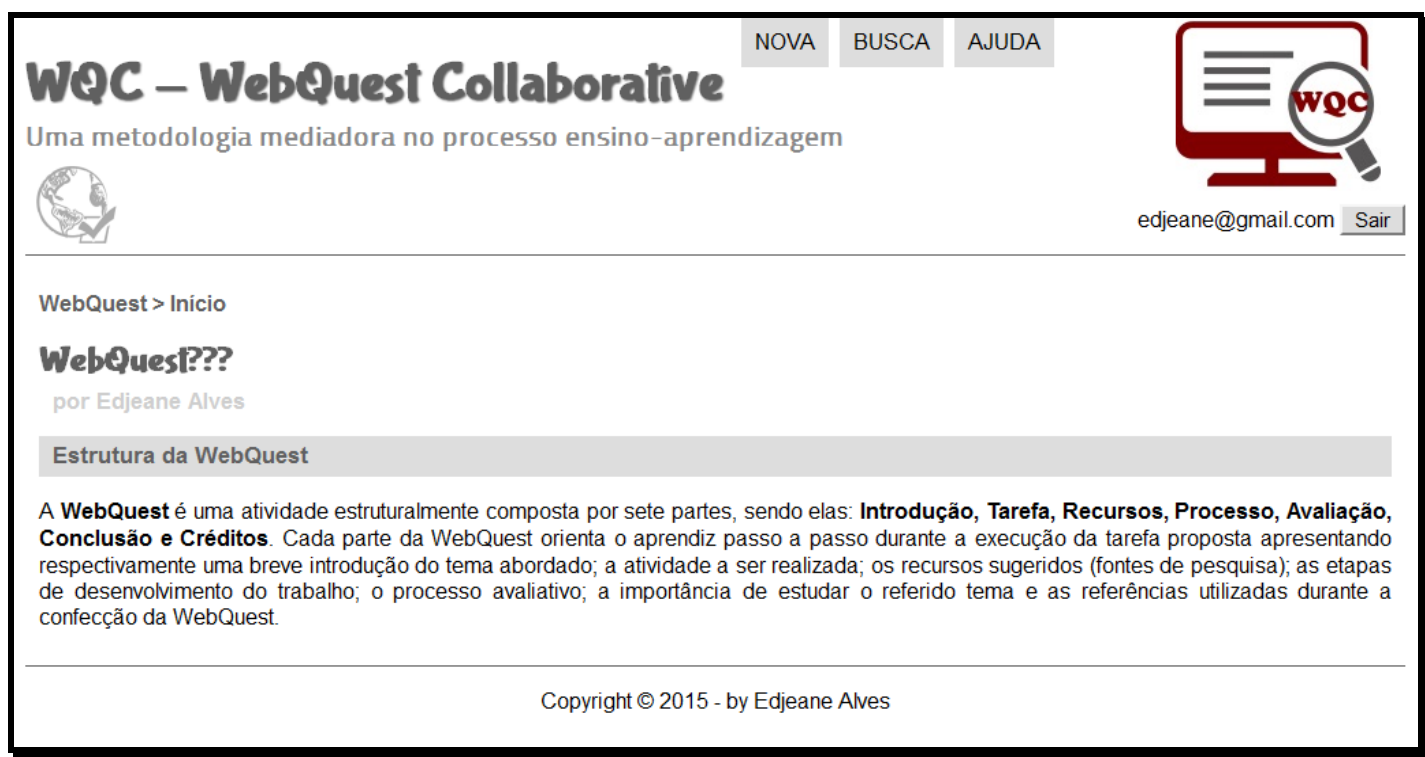

Figura 2. Tela Inicial do WQC - WebQuest Collaborative

Ao efetuar o login o usuário irá para a Tela Início na Figura 2 com o menu contendo as abas NOVA, BUSCA e AJUDA. A tela NOVA da Figura 3 permite a 
V Congresso Brasileiro de Informática na Educação (CBIE 2016)

Anais do XXVII Simpósio Brasileiro de Informática na Educação (SBIE 2016)

criação de uma nova WebQuest partindo da definição do tema a ser abordado, nessa tela há uma área para a edição do texto (semelhante às propostas no editores de texto mais comuns) e um menu lateral com a sequência das etapas da WebQuest em desenvolvimento, clicando em cada um dos números o sistema redireciona para a etapa imediatamente selecionada e esta pode ser redigida. A BUSCA leva os usuários até uma tela de consultas e a aba AJUDA fornece um breve tutorial do editor. A WebQuest produzida tem a aparência da Figura 4.

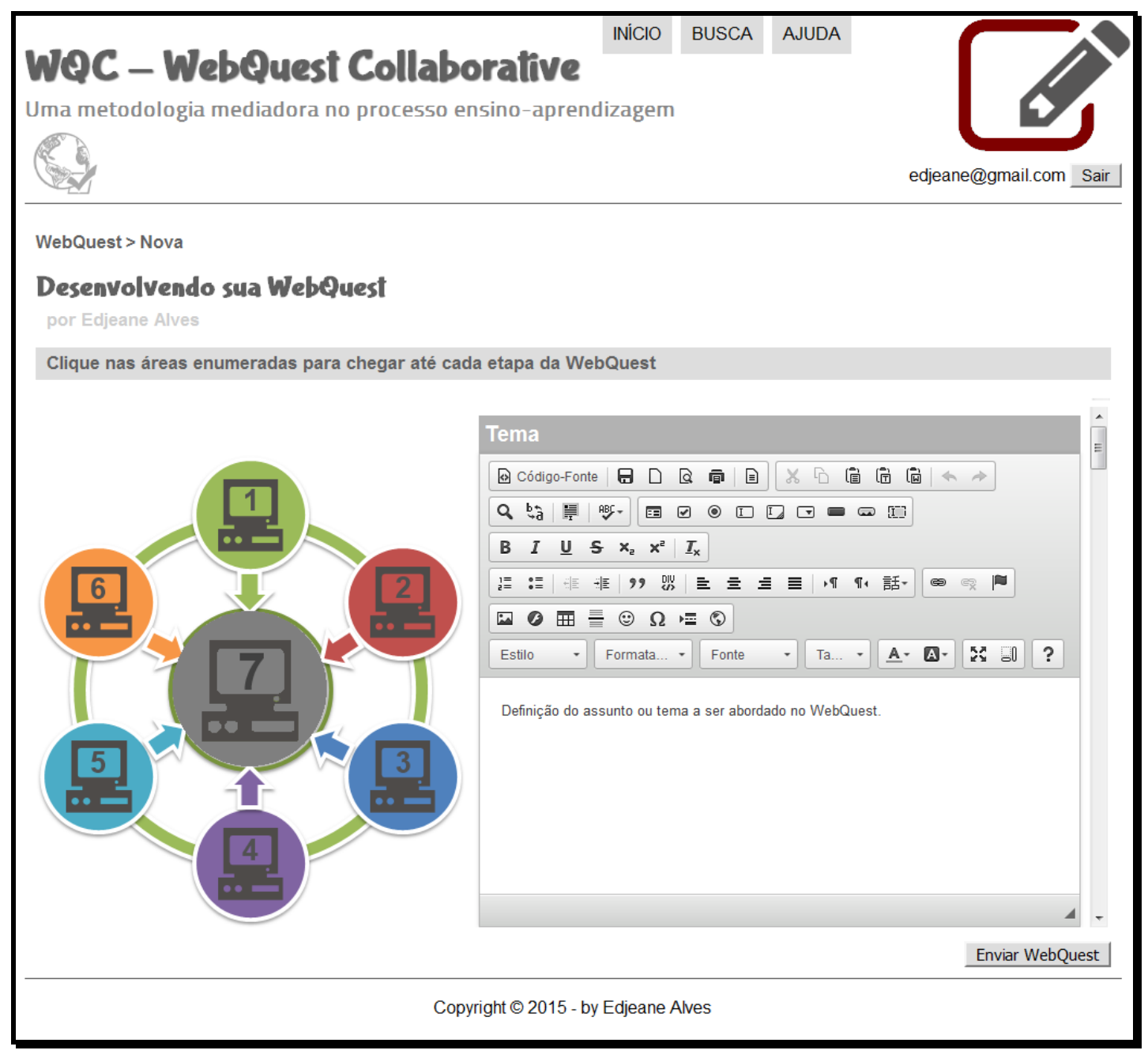

Figura 3. Tela nova do WQC - WebQuest Collaborative 
V Congresso Brasileiro de Informática na Educação (CBIE 2016)

Anais do XXVII Simpósio Brasileiro de Informática na Educação (SBIE 2016)

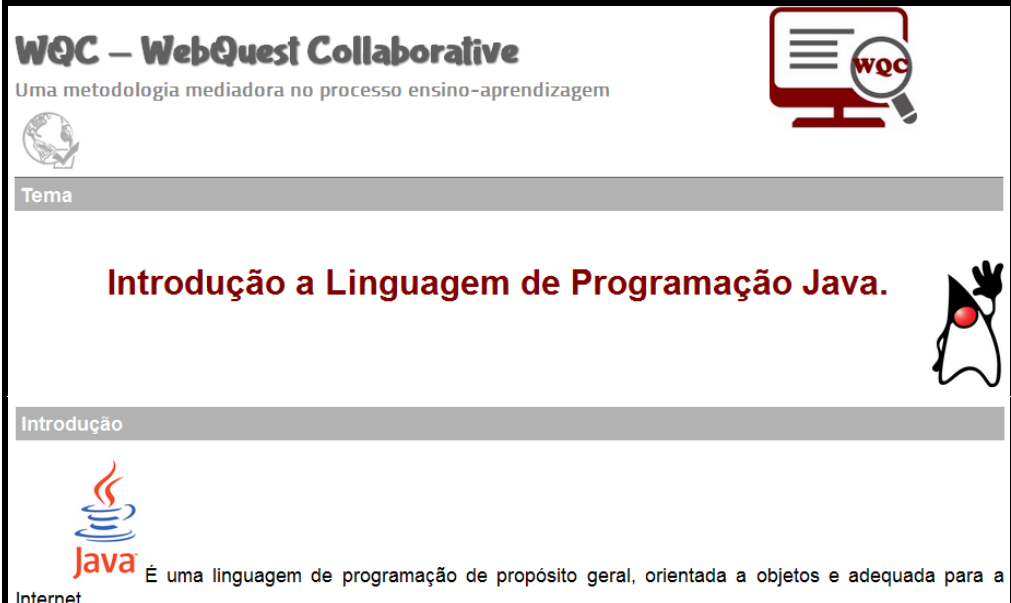
Internet.

- Utilizada para o desenvolvimento de qualquer tipo de software, com sintaxe similar ao $\mathrm{C}$

- Modelo 00 baseado em Smalltalk e Simula67;

- A linguagem JAVA foi projetada e implementada por um pequeno grupo pessoas, coordenado por James Gosling, na Sun Microsystems em Mountain View, Califórnia, em 1991.

Todas as linguagens orientadas a objetos possuem três características básicas:

- Objetos: É uma entidade lógica que contém dados e código para manipular esses dados - pode ser concreto ou um conceito (carro, cadeira, gerente), pode ser simples ou complexo, possui atributos (caractéristicas) e operações (comportamento);

- Polimorfismo: Ocorre quando uma mesma mensagem chegando a objetos diferentes provoca respostas

diferentes;

- Herança: É um mecanismo para definir novas classes a partir de classes existentes - prove reusbilidade, permite agrupar classes relacionadas de modo que elas possam ser gerenciadas de forma coletiva, Java implementa herança com o uso de extends (Java só permite herança simples).

OBS: Classes - É um tipo de dado, como os já conhecidos, para declarar variáveis, uma variável de uma classe è chamada de Objeto.

Tarefa

1) Seu primeiro Programa na Linguagem de Programação Java. - Digite e compile o programa:

public class Classe Java \{

public static void main(String [] args)

\}

System.out.println ("Hello Word!"):

2) Crie uma classe chamada Conta que representa uma conta bancária. Determine os atributos e métodos desta classe.

3) Crie uma classe chamada ContaPoupanca e outrach amada ContaCorrente, ambas subclasse de Conta. Determine os atributos e métodos específicos desta classe.

Recursos
- Apostilas;
- Vídeo-aulas;
- Livros;
- Páginas eletrônicas.
Processo
- Realizar revisão bibliográfica sugerida;
- Buscar outras fontes de pesquisa;
- Desenvolver as tarefas.
Avaliaçäo
A avaliação será realizada com base na eficiência e lógica dos programas desenvolvidos.
Conclusão
Expor uma breve introdução da Linguagem de Programação Java.
Créditos
DEITEL, H. M.; DEITEL, P. J. Java: Como Programar . 6. Ed. São Paulo: Pearson Education, 2005.
https://www.google.com.br/search?q=introdu\%C3\%A7\%C3\%A30+a+java\&newwindow=1\&client=firefox-
b\&source=Inms\&tbm=isch\&sa=X\&ved=0ahUKEwjFm5PJ5brOAhXKg5AKHa1vC4cQ_AUICigD\&biw=1366\&
bih=608\#imgrc=pzqOxfBcEb7zHM

Figura 4. Tela página WQ do WQC - WebQuest Collaborative 
V Congresso Brasileiro de Informática na Educação (CBIE 2016)

Anais do XXVII Simpósio Brasileiro de Informática na Educação (SBIE 2016)

\subsection{Requisitos e modelagem do sistema}

Foram levantados Requisitos Funcionais e Requisitos Não Funcionais para o WQC e a seguir alguns RF:

RF. 01: Cadastrar usuário - $\mathrm{O}$ editor de WQ permitirá que visitantes realizem cadastro e se tornem usuários com acesso ao sistema de forma gratuita.

RF. 02: Criar o Tema da WQ / RF. 03: Criar a etapa Introdução da WQ / RF. 04: Criar a etapa Tarefa da WQ / RF. 05: Criar a etapa Recursos da WQ / RF. 06: Criar a etapa Processo da WQ / RF. 07: Criar a etapa Avaliação da WQ / RF. 08: Criar a etapa Conclusão da WQ / RF. 09: Criar a etapa Créditos da WQ - Nestes requisitos os usuários cadastrados definem as etapas para a sua WQ criada conforme cada requisito.

RF. 10: Formatar texto da WQ - Para cada etapa todas as formatações possíveis devem estar refletidas na WQ pronta.

RF. 11: Inserir imagens na WQ / RF. 12: Formatar imagens da WQ - Imagens podem ser inseridas e formatadas na WQ.

RF. 13: Criar tabelas na WQ / RF. 14: Inserir tabelas na WQ - Tabelas podem ser criadas e inseridas na WQ.

RF. 15: Inserir hiperlinks na WQ / RF. 16: Formatar hiperlinks na WQ - Hiperlinks podem ser inseridos e formatados na WQ.

RF. 17: Editar WQ / RF. 18: Consultar WQ / RF. 19: Remover WQs - Os usuários cadastrados podem editar, consultar e remover suas WQs.

RF. 20: Encerrar sessão - Os usuários podem sair do sistema a qualquer momento.

RF. 21: Produzir WQs com todas as etapas - O editor deve ser composto pelos tópicos estruturais: introdução, tarefa, recursos, processo, avaliação, conclusão e créditos.

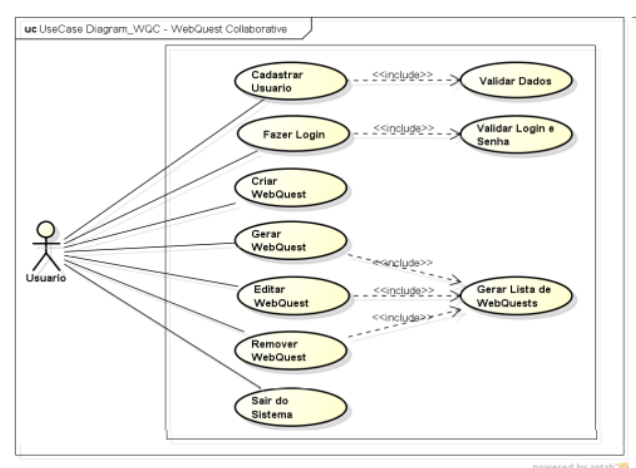

Figura 5. Diagrama de casos de uso

Para o WebQuest Collaborative foi criado o Diagrama de Casos de Uso conforme a Figura 5 e observa-se apenas um ator (usuário) interagindo com o sistema, este pode cadastrar um usuário e a partir de então, pode fazer login no sistema e criar, editar e remover WebQuests. A arquitetura do sistema está dividida em três partes (telas do sistema, modelo do sistema e base de dados), na Figura 6 é possível observar de forma detalhada as referidas divisões. A primeira parte corresponde às telas do sistema, ou seja, todos os arquivos relacionados à interface, a segunda parte trata do modelo do sistema, inclui todos os arquivos responsáveis por gerenciar a base de dados e 
V Congresso Brasileiro de Informática na Educação (CBIE 2016)

Anais do XXVII Simpósio Brasileiro de Informática na Educação (SBIE 2016)

proporcionar a execução das operações solicitadas pelo usuário e disponibilizadas pelo sistema, a terceira e última parte abrange a base de dados do sistema.

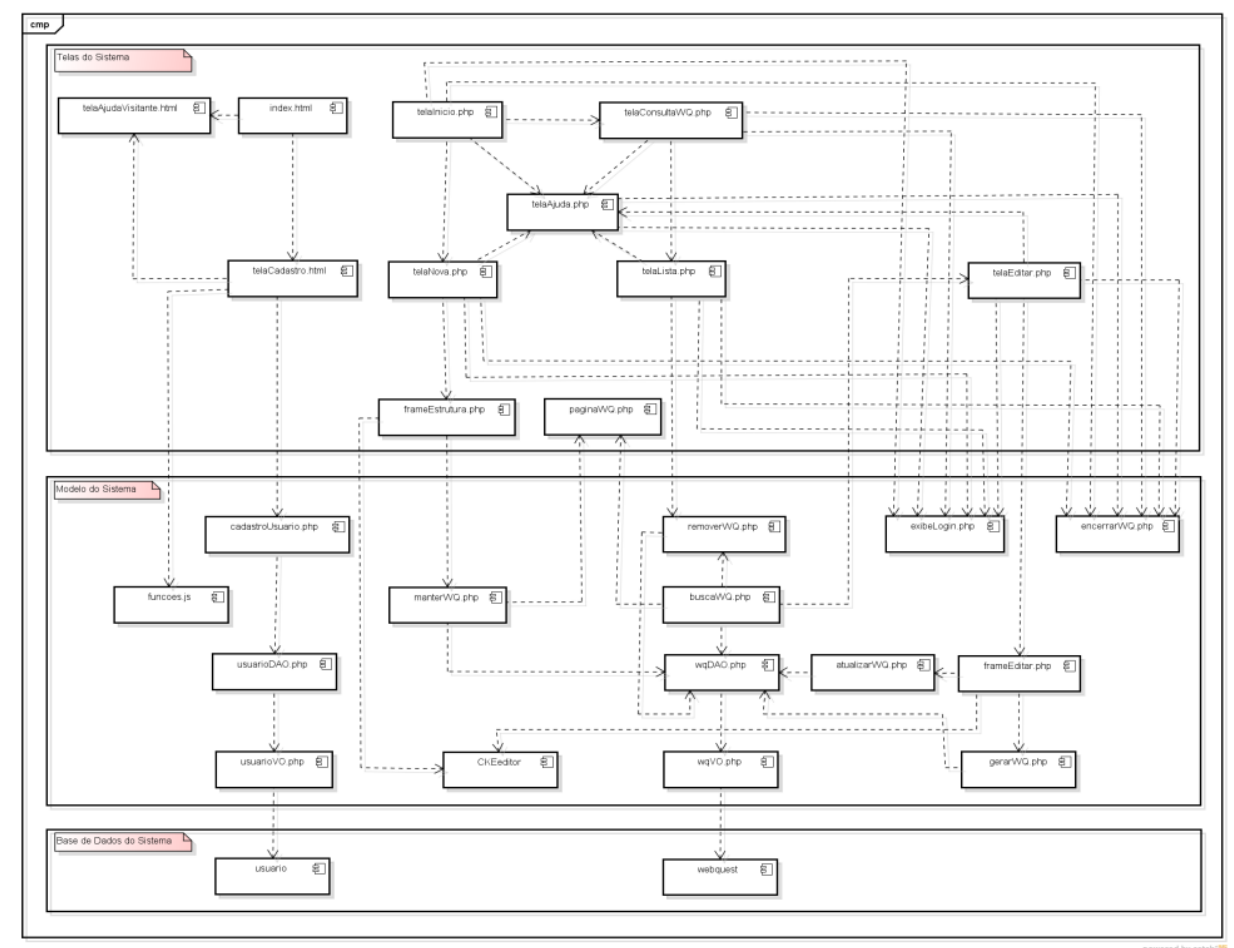

Figura 6. Diagrama de componentes

\subsection{Resultados da validação}

A validação do sistema foi realizada em uma amostra de professores composta por 1 homem e 4 mulheres, todos com o Ensino Superior completo e $60 \%$ deles pós-graduado. Os educadores lecionam Língua Portuguesa e Matemática no Ensino Fundamental II e Médio, Educação Infantil e Ensino Fundamental I. Dentre esses professores 60\% responderam que elaboram material didático extra mais de cinco vezes por semestre, $20 \%$ cinco vezes e $20 \%$ duas vezes. $40 \%$ deles utilizam computador para uso pessoal por mais de 7 horas semanais, $40 \%$ utilizam por mais de 3 horas e $20 \%$ nunca usam. Enquanto que para atividades profissionais, $40 \%$ utilizam por mais de 7 horas, $40 \%$ por 7 horas e $20 \%$ apenas 3 horas. Quando perguntados sobre a realização de pesquisas na Internet, $40 \%$ responderam que raramente, $40 \%$ com frequência e $20 \%$ diariamente. Quanto ao nível de experiência com editores de texto, 40\% acham fácil, $40 \%$ têm pouca dificuldade e $20 \%$ têm muita dificuldade. Dentre os educadores apenas $20 \%$ já criaram uma página de Internet e $80 \%$ não conheciam a metodologia WebQuest.

Em relação ao WQC, $100 \%$ dos professores da amostra disseram não sentir dificuldades durante sua utilização e consideram a aparência visual agradável de usar, inovadora e moderna. Todos os professores conseguiram concluir as etapas propostas pela WebQuest utilizando o WQC. O sistema foi bem avaliado, com fácil utilização e nenhum erro identificado durante sua execução, funcionalidade desnecessária ou limitação séria. Desse modo, $100 \%$ da amostra disseram que utilizariam o WQC para desenvolver atividades com seus alunos e sugeriram a inclusão de um corretor ortográfico no sistema para facilitar a edição dos trabalhos. 
V Congresso Brasileiro de Informática na Educação (CBIE 2016)

Anais do XXVII Simpósio Brasileiro de Informática na Educação (SBIE 2016)

\section{Conclusão}

O editor WQC - WebQuest Collaborative é uma ferramenta totalmente em português, com código aberto e livre, possui uma interface intuitiva e fácil de usar, atendendo às necessidades dos usuários e apresentando como propósito geral incentivar a criatividade do professor. O WQC disponibiliza para os educadores o meio para que estes proponham aulas diferenciadas, de forma inovadora, incrementando os mecanismos educacionais e aproximando os alunos da pesquisa, por meio da utilização de recursos providos pela Internet, conforme a metodologia WebQuest, promovendo a difusão das tecnologias de informação no ambiente escolar e por consequência trazendo consigo a atratividade através do uso desses meios.

O sistema foi validado por professores, sendo avaliado de forma positiva, com ressalva apenas em relação à ausência de um corretor ortográfico. Os educadores desenvolveram sua própria WebQuest sem maiores dificuldades e como sugestão lançaram a ideia de ser acrescentado ao software um corretor ortográfico para auxiliar durante a redação dos textos constantes no documento.

\section{Referências}

Bottentuit Junior, J. B e Coutinho, C. P. (2010) "O Uso da Estratégia WebQuest no Ensino Superior: uma análise de duas experiências”, Em: Revista Novas Tecnologias na Educação (RENOTE).

Camargo, E. Z. e Fernandes, C. T. (2010) "WQE: um Editor de WebQuests Versátil", Em: Anais do Simpósio Brasileiro de Informática na Educação.

Dodge, B. (1995) WebQuests: A technique for Internet -Based Learning. The Distance Educator. Tradução de Jarbas Novelino Barato.

Dodge, B. (2003). Webquest: uma técnica para aprendizagem na rede internet. http://www. webquest. futuro. usp. br/artigos/textos_bernie. html, p. 10-13.

IMS Global Consortium (2003) "IMS Learning Design Specifications". http://www.imsglobal.org/learningdesign/index.html, setembro.

Neto, J. S., Passaro, A. e Fernandes, C. T. (2014) "Webquest como Ferramenta de Apoio a Alunos na Aprendizagem de Sensores e Atuadores", Em: II Simpósio de Ciência e Tecnologia do IEAv.

Passerino, L. M. e Santarosa, L. M. C. (2000) "Uma visão sócio-histórica da interação dentro de ambientes computacionais", Em: Atas do V Congresso Ibero-Americano de Informática na Educação RIBIE, p. 3-4.

Silva, F. Q. e Ferrari, H. O. (2009) "A Webquest como atividade didática potencializadora da educação", Em: Revista Novas Tecnologias na Educação (RENOTE).

Vieira, Y. (2008) "Webquester: Ferramenta para Criação de Webquest com Avaliações On-Line".

Vygotsky, L. S. (1989). Pensamento e linguagem. São Paulo: Martins Fontes. 